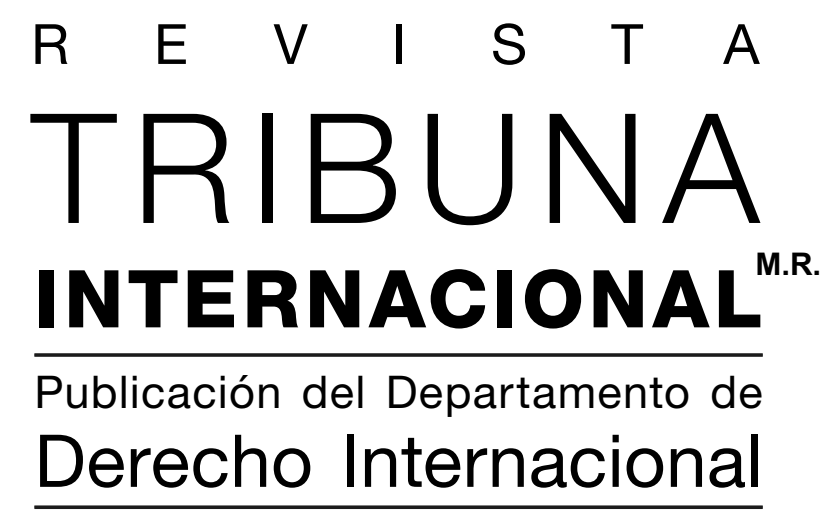

Volumen $4 / N^{\circ} 7 / 2015$

DERELTAD DE

UNIVERSIDAD DE CHILE 


\section{Rector de la Universidad de Chile}

Ennio Vivaldi Véjar

Av. Alameda Libertador Bernardo

O’Higgins 1058, Santiago

Representante legal

Davor Harasic Yaksic

Decano de la Facultad de Derecho

Universidad de Chile

Director del Departamento de

Derecho Internacional

Edmundo Vargas Carreño

Director de la Revista Tribuna

Internacional

Mario Ramírez Necochea

Editor General de la Revista Tribuna

Internacional

Luis Valentín Ferrada Walker

\section{Comité Editorial}

Íñigo Álvarez Gálvez (Universidad de Chile, Chile)

Gonzalo Aguilar (Universidad de Talca, Chile)

José Carlos Fernández Rosas

(Universidad Complutense de Madrid,

España)

Claudio Grossman (American

University, EE.UU)

Mattias Kumm (New York University,

EE.UU)

Hugo Llanos (Universidad Central, Chile)

Cecilia Medina (Universidad Diego

Portales, Chile)

Elina Mereminskaya (Universidad de Chile, Chile)

Mónica Pinto (Universidad de Buenos Aires, Argentina)

\section{Revista Tribuna Internacional M.R.}

Publicación del Departamento de Derecho Internacional de la Facultad de Derecho de la Universidad de Chile. $\mathrm{Su}$ objetivo es fomentar la reflexión, el debate, el análisis y la comunicación sobre el derecho internacional en forma pluralista y con rigor científico. Se publica cada semestre en los meses de junio y diciembre mediante convocatoria abierta a la publicación de artículos y monografías inéditos, comentarios de jurisprudencia, recensiones y comentarios de libros, en los campos de derecho internacional público y privado, derecho internacional de los derechos humanos y relaciones internacionales, tanto en castellano como en inglés.

Volumen 4/ No 7 / 2015

www.tribunainternacional.uchile.cl

ISSN 0719-210X (versión impresa)

ISSN 0719-482X (versión en línea)

Departamento de Derecho Internacional Facultad de Derecho

Universidad de Chile

Av. Santa María 076, $4^{\circ}$ piso

Providencia, Santiago de Chile

\section{Diseńo y producción:}

Gráfica LOM

www.lom.cl

Impreso en Chile/ Printed in Chile

Se autoriza la reproducción total o parcial del contenido de la publicación, siempre que se reconozca y cite el/ la/ los/ las autor/a/es/as y la publicación, no se realicen modificaciones a la obra y no se la utilice para fines comerciales. 
Revista Tribuna Internacional

Volumen $4 \cdot \mathrm{N}^{0} 7 \cdot 2015 \bullet$ pp. 129-151

ISSN 0719-210X (versión impresa)/ISSN 0719-482X (versión en línea)

\title{
El principio Nemo iudex in causa sua ante los tri- bunales internacionales. Un estado de la cuestión ${ }^{1}$
}

\author{
The Nemo iudex in causa sua principle before the international \\ tribunals. A state of the question
}

\section{Virginia Petrova Georgieva}

virginia.geor@gmail.com

Licenciada en Derecho y especialista en Derecho de la Unión Europea y en Derecho Internacional Económico por la Escuela de Derecho de la Sorbona. Maestra en Derecho por la Facultad de Derecho de la Universidad Nacional Autónoma de México, y doctoranda en Derecho Internacional del Instituto de Investigaciones Jurídicas de esa misma universidad.

Resumen: Todos los procedimientos judiciales se basan en una serie de principios generales del Derecho. Estos principios expresan los imperativos de seguridad, justicia y equidad que deben guiar el arreglo judicial de las controversias. Algunos de estos principios generales definen gran parte de las atribuciones contenciosas de un juez y garantizan el carácter objetivo de un proceso judicial. El principio Nemo iudex in causa sua constituye un principio general del Derecho, de particular importancia para el desarrollo de los procedimientos judiciales de resolución de las controversias. La independencia y la imparcialidad del juez son inherentes al ejercicio de su función judicial porque aseguran su calidad de "tercero" al litigio. El respeto de estos dos requerimientos básicos se torna fundamental para el funcionamiento de todos los tribunales tanto internos como internacionales. No obstante, los postulados del principio Nemo iudex in causa sua cobran particular relevancia para los jueces internacionales, debido al contexto específico en el que éstos desempeñan su función judicial. La cristalización del principio Nemo iudex in causa sua en los estatutos constitutivos de varias jurisdicciones internacionales y su aplicación convergente en la jurisprudencia internacional ponen de manifiesto la naturaleza común de este principio para todas las esferas especializadas de impartición de la justicia internacional.

Palabras claves: independencia, imparcialidad y jueces internacionales.

Abstract: All the judicial proceedings are based in some general principles of law. Those principles express the imperatives of security, justice and equity which have to guide the judicial settlement of disputes. Some of those general principles define an important part of the judicial function and guarantee the objective nature of a judicial process. The principle Nemo iudex in causa sua is a general principle of law, particularly important for the development of the judicial proceedings for the settlement of disputes. The independence and impartiality of a judge are inherent to the fulfilment of his judicial function as a guarantee to his quality of third party to the dispute. The observance of those two basic requirements is fundamental for the good function of all tribunals, internal and international. But the significance of the Nemo iudex in causa sua

1 Artículo enviado el 03.03.2015 y aceptado el 19.05.2015. 
principle has a special meaning for the international judges, due to the specificity of the context in which they fulfill their judicial function. The crystallization of the Nemo iudex in causa sua principle in the Constitutive Statutes of the different international judicial bodies and its convergent application in the international case-law demonstrate the common nature of this principle for all the specialized fields of the international justice.

Keywords: Independence, Impartiality, International judges.

\section{Introducción}

Los mecanismos de resolución de las controversias en el Derecho internacional han cambiado radicalmente en las últimas décadas. Durante la mayor parte de su existencia, el sistema jurídico internacional sufría notoriamente de la escasez, o incluso de la ausencia, de órganos judiciales imparciales ${ }^{2}$. En consecuencia, muchos de los litigios internacionales se resolvían por medios alternativos (incluyendo el recurso a la fuerza) y el respeto del Derecho internacional carecía de eficacia práctica. En la actualidad, esta constatación ya no es válida. Ahora vivimos en una nueva era: la de la "judicialización” del Derecho internacional ${ }^{3}$. En efecto, a partir de la década de los noventa se produjo una multiplicación sin precedente de tribunales internacionales, de tal modo que en la actualidad, en el ámbito internacional, existen más de cincuenta órganos que cumplen con funciones de naturaleza judicial o casi-judicial ${ }^{4}$.

La "judicialización" se desarrolla en un contexto marcado por la creciente especialización de las normas y de los órganos judiciales internacionales. En efecto, todos los nuevos tribunales internacionales fueron establecidos para aplicar un conjunto de normas internacionales específicas: los tribunales competentes en materia de derechos humanos aplican el Derecho internacional de los derechos humanos, los tribunales penales internacionales aplican el Derecho penal internacional, el mecanismo de solución de controversias de la Organización Mundial del Comercio (OMC) aplica el Derecho de la OMC, el Tribunal Internacional para el Derecho del Mar aplica el Derecho internacional del mar, etc. La especialización de las jurisdicciones internacionales se deriva, en primer lugar, de la naturaleza específica de las normas sustanciales que dichos órganos

2 AвI SAAB, Georges. "The Normalization of International Adjudication: Convergence and Divergence". International Law and Politics, vol. 43, 2010, pp. 1-4.

3 Cançado Trindade, Antonio Augusto. La expansión de la jurisdicción internacional y su importancia para la realización de la justicia. UN Lecture Series. [en línea] <http://legal.un.org/avl/ls/Cancado-Trindade_HR_ video_2.html> [consulta: 10 de junio del 2015].

4 Weвв, Philippa. International Judicial Integration and Fragmentation. Oxford, Oxford University Press, 2013, p. 1.

5 Petrova Georgieva, Virginia. “La ‘judicialización’: una nueva característica del orden jurídico internacional”. Anuario Mexicano de Derecho Internacional. Vol. XV, 2015, pp. 3-45. 
judiciales aplican e interpretan. Pero además, la especialización de su función judicial respectiva resulta de la especificidad de las reglas jurídicas que gobiernan sus respectivos procedimientos contenciosos. A primera vista, los estatutos constitutivos de las múltiples jurisdicciones internacionales contienen múltiples normas procesales internacionales.

A pesar de este particularismo de las normas procesales, es posible identificar importantes similitudes tanto en la concepción y redacción de las mismas como en su interpretación y aplicación concreta en la jurisprudencia de los tribunales internacionales. Es de señalarse que todos los procesos judiciales especializados que rigen en el ámbito internacional se basan en una serie de principios generales del Derecho, comunes a los órdenes jurídicos de las "naciones civilizadas". Dichos principios cardinales se consideran a menudo como "estándares procesales mínimos", "principios del proceso judicial", "normas procesales fundamentales" o "principios de justicia procesal" (principles of procedural

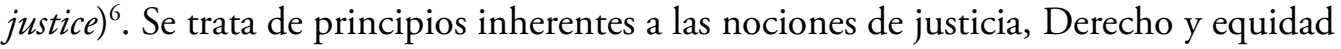
que deben guiar la resolución de los litigios internacionales. La aplicación, por parte de los tribunales internacionales, de principios procesales, establecidos objetivamente, constituye una característica definitoria de este modo de arreglo de las controversias 7 . Además, estos "principios de justicia procesal" garantizan, correlativamente, una serie de derechos procesales fundamentales, reconocidos a las partes en un procedimiento judicial internacional. No existe un acuerdo sobre las fuentes de estos principios y no se ha establecido su catálogo exacto. Por su naturaleza misma, los principios generales del Derecho, aplicables en el proceso judicial internacional, son principios comunes a los órdenes jurídicos internos de los Estados miembros de la comunidad internacional. Debido a su importancia fundamental para la óptima impartición de justica, dichos principios fueron traspuestos en el ámbito del Derecho internacional. En la mayoría de los casos se trata de principios originados en el Derecho romano y dotados de una "sabiduría jurídica" de más de 20 siglos de antigüedad.

Para los propósitos limitados de la presente investigación, analizaremos un principio general y fundamental del proceso judicial internacional: el principio Nemo iudex in causa sua. El objetivo de este análisis será comprobar las convergencias (o, en su caso, las divergencias) en la concepción, aplicación e interpretación de este principio en los esquemas especializados de resolución de las controversias ante seis tribunales internacionales: la Corte Internacional de Justicia (CIJ), el Tribunal Internacional para el Dere-

6 Gaffney, John P. "Due process in the World Trade Organization, the need for procedural justice in the dispute settlement system". American University International Law Review, vol. 14, 1999, p. 1179.

7 El arreglo judicial de las controversias internacionales se basa en un derecho procesal, objetivo y común para todos los justiciables. En otros mecanismos de solución de las controversias internacionales, como por ejemplo en el arbitraje, el derecho procesal que el tribunal ad hoc aplica es establecido, de común acuerdo, por las partes en la controversia. 
cho del Mar (TIDM), el Tribunal Penal para la ex Yugoslavia (TPIY), el Tribunal Penal Internacional para Ruanda (TPIR), la Corte Europea de Derechos Humanos (CEDH) y la Corte Interamericana de Derechos Humanos (CIDH). En primer lugar, examinaremos los dos postulados normativos de dicho principio, en lo relativo a la independencia e imparcialidad de los jueces internacionales. En segundo lugar, nos interesaremos en la cristalización del principio Nemo iudex in causa sua en los estatutos constitutivos de los tribunales internacionales y pondremos de manifiesto su interpretación y aplicación convergente en la jurisprudencia de los mismos. Finalmente, realizaremos algunas conclusiones de este análisis comparativo para abordar la función de este principio general en el actual funcionamiento de los mecanismos judiciales de impartición de justicia en el ámbito del Derecho internacional.

\section{La doble exigencia del principio Nemo iudex in causa sua: La independencia y la imparcialidad de los jueces internacio- nales}

El principio general Nemo iudex in causa sua proviene del adagio latín Nemo debet esse iudex in propria causa sua, quia no potest case iudex et pars (Nadie debe ser juez de su propia causa porque no se puede ser (a la vez) juez y parte.) Dos otras versiones de dicho principio se expresan en las máximas: Ne quis in sua causa iudicet vel sibi ius dicat (Nadie debe examinar su propia causa como un juez o hacerse un juicio a sí mismo) y Nemo potest ese simul actor et iudex (Nadie puede ser parte y juez al mismo tiempo) ${ }^{8}$.

El término causa, que puede utilizarse como sinónimo de res, tiene múltiples significados en el latín y en el Derecho Romano. En el contexto de las máximas anteriormente señaladas, su significado se relaciona con la organización judicial y lo referente a un “juicio". El objetivo principal de estas tres máximas consiste en subrayar el rechazo de toda asimilación entre los jueces y las partes en un juicio. Además, dichas reglas establecen la prohibición para los jueces de involucrarse personalmente en los casos que deben resolver de manera objetiva.

La máxima Nemo iudex in causa sua expresa un principio general del Derecho, común a los órdenes jurídicos de los Estados miembros de la comunidad internacional. En los sistemas pertenecientes a la familia jurídica romano-germánica, dicho principio forma parte de las reglas que gobiernan el ejercicio mismo de la función judicial. En los

8 Swartzenberger, Georg. "The Nemo Iudex in Sua Causa Maxim In International Judicial Practice”. AngloAmerican Law Review, 1972, p. 482. 
Revista Tribuna Internacional

Volumen $4 \cdot \mathrm{N}^{0} 7 \cdot 2015 \cdot$ pp. 129-151

ISSN 0719-210X (versión impresa)/ISSN 0719-482X (versión en línea)

órdenes jurídicos de Common Law, el principio Nemo iudex in causa sua es uno de los componentes básicos de la doctrina de Justicia Natural (Natural Justice) ${ }^{9}$.

El principio Nemo iudex in causa sua fundamenta dos requerimientos imperativos, inherentes a la noción misma de Derecho y de justicia: la independencia y la imparcialidad del juez. La impartición de la justicia y el acto de juzgar tienen que ser confiados a un tercero, independiente e imparcial, que decida de manera equitativa y neutral la resolución de un litigio. El arreglo judicial de las controversias es destinado a promover el respeto del Derecho por encima de los intereses particulares. De ahí que la noción misma de justicia se relaciona con la independencia e imparcialidad de los órganos que la imparten. En este sentido, las principales representaciones simbólicas de la justicia enfatizan su necesaria neutralidad y carácter objetivo ${ }^{10}$.

La independencia e imparcialidad de los jueces los define como tales porque garantizan su calidad (inherente) de "terceros" a la causa del litigio. Dichas exigencias son, por lo tanto, uno de los elementos que definen a las nociones de juez, justicia y jurisdicción, tanto en el ámbito interno como en el internacional ${ }^{11}$.

El derecho a un tribunal independiente e imparcial constituye, además, un derecho humano básico, garantizado en todos los instrumentos internacionales de protección de los derechos humanos ${ }^{12}$. Así por ejemplo, el artículo 10 de la Declaración universal de derechos humanos prevé que: "Toda persona tiene derecho, en condiciones de plena igualdad, a ser oída públicamente y con justicia por un tribunal independiente e imparcial, para la determinación de sus derechos y obligaciones o para el examen de cualquier

9 Dicha doctrina se refiere a la necesidad de impartir la justicia de manera natural, es decir conforme con los principios de la naturaleza, basados en la igualdad y la equidad. La Natural Justice se basa en dos máximas del Derecho Romano: nemo iudex in causa sua (nadie debe ser juez en su propia causa) y audi alteram partem (nadie debe ser condenado sin ser escuchado previamente).

10 En este sentido, a partir de la Revolución Francesa la diosa romana Iustitia se empieza a representar con los ojos vendados para marcar así su carácter objetivo, estrictamente apegado a la ley. Cabe señalar que ni las representaciones en Roma de la diosa Iustitia, ni las estatuas griegas de la diosa Temis contaban con este detalle. Como lo afirma Emmanuelle Jouannet, la justicia, a lo largo de la historia, fue representada de dos maneras distintas: la primera imagen, y la más conocida, es la de una mujer con los ojos vendados sosteniendo en sus manos los dos platos de una balanza equilibrada, y la segunda, mucho menos conocida, es la de un ojo gigantesco (iustitiae oculus) abierto sobre el mundo. Estas dos imágenes revelan la paradoja de la justicia: por un lado ésta debe ser neutral y libre de todo vínculo con el mundo que la rodea y, por otra, debe ser capaz de ver todo, incluso lo más oculto (JouAnNET, Emmanuelle. "Actualité des questions d'indépendance et d'impartialité des juridictions internationals: la consolidation d'un tiers pouvoir international?", en RuIz-FABri, Hélene y Sorel, Jean-Marc (dir.). L'indépendance et l'impartialité des juridictions internationals. París, Editorial Pedone, 1999, p. 271).

11 Jounnnet, “Actualité des questions...", op.cit., p. 271.

12 Dicho derecho es, en particular, protegido por el artículo 10 de la Declaración Universal de derechos humanos, el artículo 14 del Pacto Internacional sobre derechos civiles y políticos, el artículo 6 de la Convención europea de derechos humanos, el artículo 8 de la Convención interamericana de derechos humanos y por el artículo 7 de la Carta africana de los derechos humanos y de los pueblos. 
acusación contra ella en materia penal". De la misma manera, el artículo 6-1 de la Convención europea de derechos humanos establece que: "Toda persona tiene derecho a que su causa sea oída equitativa, públicamente y dentro de un plazo razonable por un tribunal independiente e imparcial, establecido por ley (...).” Este derecho humano constituye una de las garantías fundamentales del debido proceso (due process of law) ${ }^{13}$, cuyo objetivo principal consiste en garantizar el respeto de los derechos individuales en la impartición de la justicia. Como lo indica la Asamblea General de la ONU en varias de sus resoluciones: el respeto del derecho y la correcta impartición de la justicia desempeñan una función central en la promoción y protección de los derechos humanos ${ }^{14}$.

El principio Nemo iudex in causa sua, como fundamento del derecho humano a ser juzgado por un tribunal independiente e imparcial, suele considerarse como dirigido a los jueces nacionales y no internacionales. En relación con esto, se han adoptado varios instrumentos internacionales que establecen estándares en la materia para los miembros de los tribunales internos ${ }^{15}$.

La multiplicación de los tribunales internacionales atrajo la atención sobre la aplicación del principio Nemo iudex in causa sua en la práctica judicial internacional. En particular, el respeto de dicho principio fue considerado como uno de los elementos que aseguran la credibilidad y legitimidad de la función judicial, ejercida por los numerosos tribunales internacionales ${ }^{16}$. La independencia e imparcialidad de los jueces internacionales es vista como un elemento de la "juridización” de la resolución de las controversias in-

13 Aunque muchas veces se considera que el debido proceso tiene su origen en la Carta Magna inglesa de 1215, dicho concepto es un término utilizado principalmente en el Derecho estadounidense. En efecto, la Quinta Enmienda a la Constitución americana dispone que: "no person shall be (...) deprived of life, liberty or property without due process of law (...)". El contenido normativo de la noción de debido proceso varía en función de las circunstancias específicas de cada caso particular. En el Common Law, dicho concepto muchas veces se considera como análogo a los principios de Natural justice, mientras que en los sistemas romanogermánicos, en particular en el francés, se le considera como parte de los droits de la défense (derechos de la defensa). En el ámbito internacional, el debido proceso agrupa una serie de principios generales del Derecho, comunes a todos los sistemas de impartición de justicia. Dichos principios son principalmente los derivados de las máximas nemo iudex in causa sua y audi alteram partem. Otro importante componente del debido proceso es el principio de igualdad de las partes en un procedimiento contencioso. Dicho principio implica, entre otros, el derecho de acceso a la justicia o derecho al juez (droit au juge), las garantías procesales de composición del tribunal, el secreto de las deliberaciones o el derecho a una sentencia motivada (GAFFNEY, "Due process in...", op.cit., pp. 1176-1177).

14 En particular, en las resoluciones 50/180 del 22 de septiembre de 1995 y 48/137 del 20 de diciembre de 1993, intituladas Derechos humanos en la impartición de la justicia.

15 Entre las iniciativas más importantes podemos citar: los Principios básicos de las NU relativos a la independencia de la Judicatura de 1985, [en línea] <http://www2.ohchr.org/spanish/law/judicatura.htm> [consulta: 10 de junio del 2015]; el Código de estándares minimos de independencia judicial de la International Bar Assotiation de 1983, los Principios de Beijing sobre la independencia de la judicatura de 1995 y los Principios de Siracusa de 1981.

16 Mackenzie, Ruth y Sands, Philippe. "International Courts and Tribunals and the Independence of the International Judge”. Harvard International Law Journal, vol. 44, 2003, p. 274. 
ternacionales. Este renovado interés por la aplicación de dicho principio en el Derecho internacional, condujo a la adopción de varias propuestas de "estándares comunes" en materia de independencia e imparcialidad de los jueces internacionales ${ }^{17}$.

Muchas veces parece difícil distinguir la independencia de la imparcialidad de un juez. En términos generales, tanto la independencia como la imparcialidad permiten al juez definirse como un "tercero" respecto a las partes y respecto a la controversia que se le somete. Si el juez no es parte, entonces no será parcial (partial en inglés y en francés), sino imparcial (impartial en inglés y en francés). De igual modo, si el juez es un "tercero", éste no debe ser dependiente de influencias (políticas) externas, porque para ser un verdadero "tercero", el juez debe permanecer autónomo y libre de toda presión o tentativa de subordinación ${ }^{18}$.

Una parte de la doctrina considera que la imparcialidad es una noción incluida en el concepto más genérico de independencia y se refiere a la independencia personal de un juez vis-à-vis de una o de las dos partes en un litigio ${ }^{19}$. Para otros autores, dichos principios deben ser distinguidos ${ }^{20}$. De acuerdo a un método de análisis propuesto por el profesor Shimon Shetreet en 1976, la independencia de los jueces se subdivide en independencia individual e independencia colectiva. La individual sería relativa, por ejemplo, a la permanencia del juez en el puesto o a su imparcialidad, y la independencia colectiva, de todo el órgano judicial, se basaría en aspectos institucionales, como por ejemplo su relación con los órganos políticos que lo establecieron o que deciden su presupuesto.

En términos generales, la independencia se refiere a la ausencia de influencias del exterior. Según el diccionario Salmon, independencia significa "el hecho por una persona o por una entidad de no depender de una autoridad, otra que la suya propia”. Un tribunal sería, por tanto, independiente si no se encuentra bajo la autoridad de otro(s). La independencia no significa libertad completa. Una jurisdicción no puede ser completamente libre, sino que permanece sujetada a varias limitaciones. No obstante, ésta no

17 El más importante intento de sistematizar las reglas relativas a la independencia e imparcialidad de los tribunales internacionales son los Principios de Burgh House relativos a la independencia de la judicatura internacional, elaborados por un grupo de renombrados expertos en derecho internacional en Burgh House, Inglaterra, en el año 2004.

18 Jounnnet, "Actualité des questions...", op.cit., p. 277.

19 Por ejemplo, Treschel, Stefan. Human Rights in Criminal Proceedings. Oxford, Oxford University Press, 2005, p. 45; Miller, Nathan. Burgh House Principles on the independence of the international judiciary: General overview of the issue. Background Paper for the Meeting of the Study Group of the International Law Association. Londres, Burgh House, Hampstead, 2 de febrero del 2002, p. 3. [en línea] <http://www.pictpcti.org/activities/ILA_study_grp/ILA1.pdf> [consulta: 10 de junio del 2015].

20 VAN DIJK, Peter. The right of an accused to a fair trial under international law. Leiden, Kluwer Law International, 1998, p. 498. 
debe subordinarse a otros poderes ni someterse a presiones exteriores. La independencia garantiza así un "libre juicio", indispensable para la buena impartición de la justicia"

Por su parte, la imparcialidad se refiere a los prejuicios o tendencias que un juez puede tener respecto a una de las partes en un caso concreto. La imparcialidad traduce violaciones "particulares" del principio Nemo iudex in causa sua, mientras que la independencia revela limitaciones institucionales o sistémicas al ejercicio neutro de la función judicial. Como lo afirmó el TPIR en el caso Kanyabashi22, "la independencia judicial connota la libertad respecto a presiones e interferencias externas. La imparcialidad es caracterizada por la objetividad al poner en balance los intereses legítimos que están en juego" ${ }^{23}$. Un juez puede ser independiente, pero a la vez completamente parcial en la resolución de un litigio. No obstante, el juez sólo puede ser imparcial si es suficientemente independiente. En el Dictionnaire de Droit International Public se enuncia que la imparcialidad de los jueces, árbitros, expertos o cualquier otra persona que desempeńa una función similar, significa "no tomar partido, no tener prejuicios ni conflictos de interés” respecto a las partes o respecto a la cuestión jurídica que se debe resolver ${ }^{24}$. La imparcialidad se referiría entonces a una "justa distancia”, cuyos contornos precisos son muy difíciles de definir.

Todos los tribunales internacionales están sujetos al respeto del principio Nemo iudex in causa sua. La independencia e imparcialidad de los jueces que sesionan en las jurisdicciones internacionales son exigencias básicas del ejercicio de su función judicial. Su observancia es garantizada, en primer lugar, por la existencia de normas convencionales que consagran (o cristalizan) este principio general del Derecho. En segundo lugar, el respeto de dicho principio puede deducirse de la práctica jurisprudencial de los órganos judiciales internacionales. Tanto las trasposiciones normativas del principio Nemo iudex in causa sua, como su aplicación concreta por parte de los tribunales internacionales demuestran el carácter general y universal de dicho principio. Además, las convergencias que pueden constatarse en la aplicación jurisprudencial del principio Nemo iudex in causa sua permiten observar el desarrollo de estándares jurídicos comunes, relativos a la independencia e imparcialidad de los miembros de la judicatura internacional.

\footnotetext{
JOUANnEt, “Actualité des questions...”, op.cit., p. 283.

TPIY (1995). Prosecutor v. Kanyabashi.

TPIY (1995). Prosecutor v. Kanyabashi, párr. 35.

JouAnnet, "Actualité des questions...”, op.cit., p. 291.
} 


\section{La cristalización del principio Nemo iudex in causa sua en los estatutos constitutivos de las jurisdicciones internacionales}

Todos los estatutos constitutivos de los tribunales internacionales, objeto del estudio, contienen disposiciones que consagran expresamente el principio Nemo iudex in causa sua en su primera vertiente, relativa a la imparcialidad de los jueces internacionales.

El primer tipo de disposiciones que buscan garantizar la imparcialidad de los jueces internacionales establecen una serie de actividades incompatibles con el ejercicio de la función judicial.

Así por ejemplo, los artículos 16,17 y $24^{25}$ del Estatuto de la CIJ se refieren a los casos en los que un cargo (previa o actualmente) asumido por un juez internacional puede limitar su imparcialidad para resolver un asunto particular. Si tal fuera el caso, el juez en cuestión deberá negarse a participar en la resolución de la controversia y proceder a su recusación. Dichas disposiciones muchas veces son tomadas como ejemplo para la redacción de disposiciones similares en los estatutos constitutivos de las demás jurisdicciones internacionales. La incompatibilidad de algunos cargos internacionales con el ejercicio de la función judicial constituye una garantía a la imparcialidad de todos los jueces internacionales. Dicha garantía es sin duda una prueba del carácter común del principio Nemo iudex in causa sua para todos los tribunales internacionales.

En particular, dicha incompatibilidad se establece en los artículos $7^{26}$ y $8^{27}$ del Estatuto

25 El artículo 16 establece que: “1. Ningún miembro de la Corte podrá ejercer función política o administrativa alguna, ni dedicarse a ninguna otra ocupación de carácter profesional. 2. En caso de duda, la Corte decidira’”. De acuerdo al artículo 17: “1. Los miembros de la Corte no podrán ejercer funciones de agente, consejero o abogado en ningún asunto. 2. No podrán tampoco participar en la decisión de ningún asunto en que hayan intervenido anteriormente como agentes, consejeros o abogados de cualquiera de las partes, o como miembros de un tribunal nacional o internacional o de una comisión investigadora, o en cualquier otra calidad. 3. En caso de duda, la Corte decidirá”. Por su parte, el artículo 24 prevé la obligación de un juez de la Corte de recusarse si por algún motivo se estima incompetente para participar en los deliberados.

26 En virtud de esta disposición: "1. Los miembros del Tribunal no podrán ejercer función política o administrativa alguna, ni tener una vinculación activa con ninguna empresa que intervenga en la exploración o la explotación de los recursos del mar o de los fondos marinos o en otra forma de aprovechamiento comercial del mar o de los fondos marinos, ni tener un interés financiero en dichas empresas. 2. Los miembros del Tribunal no podrán ejercer funciones de agente, consejero ni abogado en ningún asunto. 3 . En caso de duda sobre estas cuestiones, el Tribunal decidirá por mayoría de los demás miembros presentes".

27 El artículo 8 prevé lo siguiente: "1. Los miembros del Tribunal no podrán conocer de ningún asunto en que hayan intervenido anteriormente como agentes, consejeros o abogados de cualquiera de las partes, como miembros de un tribunal nacional o internacional o en cualquier otra calidad. 2. Si, por alguna razón especial, un miembro del Tribunal considera que no debe conocer de un asunto determinado, lo hará saber al Presidente del Tribunal (...)”. 
del TIDM. Cabe señalar la similitud en la redacción de dichos artículos con lo previsto al respecto en el Estatuto de la CIJ. En el mismo sentido, la imparcialidad de los jueces de la CEDH también es garantizada a través de la prohibición de asumir algunos cargos, considerados como incompatibles con el ejercicio de la función judicial. Dicha garantía es expresada, en particular, en el artículo 4 del Reglamento de la Corte ${ }^{28}$. Por su parte, el artículo 18 del Estatuto de la $\mathrm{CIDH}^{29}$ garantiza la imparcialidad de los jueces mediante una regulación de los cargos incompatibles con el ejercicio de su función judicial. Dicha disposición es redactada en términos muy similares con lo establecido en los estatutos de los tribunales internacionales, previamente examinados. Además, el Estatuto de la $\mathrm{CIDH}$ contiene una disposición original destinada a evitar los conflictos de interés que los jueces de la Corte podrían tener con los miembros de su familia ${ }^{30}$. Las garantías a la imparcialidad de los jueces internacionales, como expresión directa del principio general Nemo iudex in causa sua, también se aplican a los miembros de los tribunales penales internacionales. En efecto, los instrumentos convencionales que regulan la actividad judicial del TPIY, del TPIR y de la CPI contienen disposiciones muy similares respecto a la necesaria imparcialidad de los jueces que los componen. En el caso de las dos jurisdicciones penales ad hoc, se trata de disposiciones casi idénticas ${ }^{31}$.

28 Tras su última modificación en febrero de 2012, el artículo 4 del Reglamento de la CEDH establece que “(...) ningún juez debe ejercer una actividad política, administrativa o profesional, incompatible con su independencia e imparcialidad, o con las demandas del ejercicio del oficio judicial a tiempo completo. Asimismo, cada juez debe declarar al Presidente de la Corte cualquier actividad adicional. En caso de desacuerdo entre el Presidente y el juez, la cuestión será llevada al pleno de la Corte (...)”.

29 En virtud de dicho artículo: "1. Es incompatible el ejercicio del cargo de juez de la Corte Interamericana de Derechos Humanos con el de los cargos y actividades siguientes: a. los de miembros o altos funcionarios del Poder Ejecutivo; quedan exceptuados los cargos que no impliquen subordinación jerárquica ordinaria, así como los de agentes diplomáticos que no sean Jefes de Misión ante la OEA o ante cualquiera de sus Estados miembros; b. los de funcionarios de organismos internacionales; c. cualesquiera otros cargos y actividades que impidan a los jueces cumplir sus obligaciones, o que afecten su independencia, imparcialidad, la dignidad o prestigio de su cargo (...)”.

30 En efecto, su artículo 18 establece que: "1. Los jueces estarán impedidos de participar en asuntos en que ellos o sus parientes tuvieren interés directo o hubieran intervenido anteriormente como agentes, consejeros o abogados, o como miembros de un tribunal nacional o internacional, o de una comisión investigadora, o en cualquier otra calidad, a juicio de la Corte. 2. Si alguno de los jueces estuviere impedido de conocer, o por algún motivo calificado considerare que no debe participar en determinado asunto, presentará su excusa ante el Presidente (...)”.

31 Según la regla 15 de las Reglas sobre procedimiento y pruebas del TPIY, un juez no puede comparecer en un juicio (de primera instancia o de apelación) en el que tiene un interés personal o algún otro tipo de relación que podría afectar su imparcialidad. En cualquier situación de este tipo el juez deberá recusarse y el Presidente del TPIY nombrará otro magistrado que lo sustituya en el cargo. En el mismo sentido la regla 15 de las Reglas sobre procedimiento y pruebas del TPIR prevé un procedimiento de recusación idéntico al establecido en las Reglas del TPIY. La única diferencia entre los dos se refiere al órgano, encargado de decidir sobre la recusación de un juez por causa de imparcialidad. En el caso del TPIY, dicha facultad es conferida al Presidente, mientras que en el caso del TPIR las demandas de recusación son presentadas al Bureau del tribunal. 
Revista Tribuna Internacional

Volumen $4 \cdot \mathrm{N}^{0} 7 \cdot 2015 \cdot$ pp. 129-151

ISSN 0719-210X (versión impresa)/ISSN 0719-482X (versión en línea)

En conclusión, podemos considerar que la regulación de los cargos incompatibles figura en todos los estatutos constitutivos de los tribunales internacionales. Además, su redacción es muy similar y los procedimientos previstos manifiestan varias analogías. Dicha regulación constituye la cristalización del primer componente del principio Nemo iudex in causa sua.

Como fue mencionado anteriormente, el segundo aspecto de dicho principio -el de la independencia de los jueces internacionales- se relaciona con factores externos a la resolución de un caso concreto. Mientras que la imparcialidad es una cuestión concerniente a la relación entre los jueces y las partes en una controversia, la independencia se refiere a la relación de los magistrados con terceros ${ }^{32}$. Las garantías que el Derecho internacional ofrece a la independencia de los jueces son numerosas y complejas. El principio de independencia de los jueces internacionales se declina en varias formas y modalidades de aplicación según la materia contenciosa y según la jurisdicción en cuestión. Así, la independencia puede ser externa cuando se trata de las relaciones entre el tribunal y sus principales justiciables, e interna cuando se examinan las garantías ofrecidas a los jueces al interior del órgano judicial. En la primera categoría de reglas se incluyen las relativas a la elección de los jueces internacionales, a sus privilegios e inmunidades, al secreto de sus deliberaciones, a las condiciones de su permanencia en el puesto, a sus remuneraciones y a los procedimientos disciplinarios de los cuales pueden ser objeto. En la segunda categoría de disposiciones (independencia del tribunal como un todo) pueden incluirse las relativas a su presupuesto general ${ }^{33}$.

Examinar la totalidad de los componentes del principio de independencia de los jueces internacionales excede los propósitos limitados de la presente investigación. Por lo tanto, centraremos nuestra atención sobre un aspecto particular de dicho principio: el de la nacionalidad de los jueces internacionales.

Para no ser juez de su propia causa (Nemo iudex in causa sua) es preciso ser independiente tanto respecto a las partes, como respecto a los terceros en una controversia. A diferencia de los árbitros internacionales, los jueces internacionales resuelven controversias en las que necesariamente participa una parte estatal. Dicho en otros términos, los jueces internacionales juzgan a los Estados soberanos, en su calidad de miembros de la comunidad internacional. Podría parecer contrario al principio Nemo iudex in causa sua que los justiciables designen a su propio juez y que éste sea en la mayoría de los casos uno de sus nacionales. De acuerdo al principio establecido en la famosa sentencia Nottebohm ${ }^{34}$, la nacionalidad siempre supone la existencia de un vínculo efectivo entre el ciudadano y su Estado nacional. ¿Cómo garantizar que en el ejercicio de las funciones judiciales, el juez no se sentirá influenciado por este vínculo?

Miller, Burgh House Principles..., op.cit., pp. 7-10.

Miller, Burgh House Principles..., op.cit., pp. 13-21.

CIJ (1955). Caso Nottebohm (Guatemala c. Lichtenstein). 
Aunque la redacción de las reglas relativas a la designación de los jueces internacionales difiere, su contenido es sumamente convergente. En general, en el Derecho internacional se combinan dos fases de designación: una nominación nacional (un Estado nomina a una persona para ser juez en una jurisdicción internacional) y una elección internacional (el nominado por su Estado nacional es posteriormente electo a través de un procedimiento internacional). Por lo tanto, es de estimarse que todos los jueces internacionales son nacionales de los Estados miembros de la comunidad internacional. Por lo general, los miembros de los tribunales internacionales tienen la nacionalidad de los Estados miembros de la organización que los creó o de los Estados que hayan ratificado sus estatutos constitutivos.

Los estatutos de casi todos los tribunales internacionales adoptan reglas muy similares respecto a la nacionalidad de los jueces que los componen. "El enfoque tradicional" retoma lo establecido en la materia a partir de la creación de la CPJI ${ }^{35}$. En efecto, en el estatuto de la primera jurisdicción internacional permanente, la cuestión relativa a la nacionalidad de los jueces se basaba, en primer lugar, en la limitación del número de jueces de la misma nacionalidad. En segundo lugar, dicho estatuto preveía la nominación de un juez ad hoc de la nacionalidad de uno de los Estados, parte en la controversia, cuando dicho Estado no contaba con un representante en la Corte al surgir el litigio ${ }^{36}$. En efecto, la presencia de dos jueces nacionales de un Estado en la composición de la Corte era visto, por los redactores de su estatuto, como susceptible de generar una duda legítima respecto a su independencia. Para contrarrestar esta duda se instituyó la figura del juez ad hoc. Los redactores del Estatuto de la CPJI consideraron que, para respetar el principio de igualdad de las armas ${ }^{37}$, los dos Estados partes en la controversia debían contar con un juez de su nacionalidad ${ }^{38}$.

Dicha regla es retomada explícitamente en los artículos $3^{39}$ y 31 del Estatuto de la CIJ ${ }^{40}$.

35 Dannenbaum, Tom. "Nationality and the international judge: The nationalist presumption governing the international judiciary and why it must be reversed?". Cornell International Law Journal, vol. 45, 2012, pp. 89-90.

36 Artículos 2 y 31 del Estatuto de la CPJI.

37 Como lo afirma la CIDH, la institución del juez ad hoc tiene por objetivo principal garantizar el principio de igualdad de las armas en la solución judicial de una controversia (Opinión consultativa OC-20/09 solicitada por la República Argentina, 29 de septiembre de 2009). Cabe señalar que la presencia de un juez ad hoc en el ámbito de la CIDH se refiere sólo a los casos que oponen un Estado a otro Estado miembro de la Convención interamericana de derechos humanos y no se aplica para los casos en los que intervienen individuos.

, Nationality and the international judge..., op.cit., p. 114

39 Según el primer párrafo del artículo 3: "1. La Corte se compondrá de quince miembros, de los cuales no podrá haber dos que sean nacionales del mismo Estado".

40 Según el artículo 31 del Estatuto de la CPJI: "2. Si la Corte incluyere entre los magistrados del conocimiento uno de la nacionalidad de una de las partes, cualquier otra parte podrá designar a una persona de su elección para que tome asiento en calidad de magistrado. (...) 3. Si la Corte no incluyere entre los magistrados del conocimiento ningún magistrado de la nacionalidad de las partes, cada una de éstas podrá designar uno de acuerdo con el párrafo 2 de este artículo". 
Revista Tribuna Internacional

Volumen $4 \cdot \mathrm{N}^{0} 7 \cdot 2015 \cdot$ pp. 129-151

ISSN 0719-210X (versión impresa)/ISSN 0719-482X (versión en línea)

Esta doble exigencia respecto a la nacionalidad de los jueces es expresada en los mismos términos en los estatutos del TIDM ${ }^{41}$ y de la $\mathrm{CIDH}^{42}$. En otros casos, dicha regla sufre algunas derivaciones de su formulación inicial. Así por ejemplo, los estatutos de las tres jurisdicciones penales internacionales (los dos tribunales penales ad hoc y la CPI) consagran el principio de limitación del número de jueces de la misma nacionalidad, pero no retoman el relativo a la institución de un juez ad hoc ${ }^{43}$. Por su parte, el Protocolo 11 de reforma a la Convención europea de derechos humanos suprimió el artículo 38 que prohibía la nominación de dos jueces de la misma nacionalidad. No obstante, las reglas que gobiernan la nominación y la elección de los jueces de la $\mathrm{CEDH}$ excluyen tal hipótesis ${ }^{44}$. En tanto, el artículo 22 de la Convención europea de derechos humanos establece un principio similar al de la designación de un juez ad hoc.

Las reglas relativas a la nacionalidad de los jueces internacionales buscan evitar todo tipo de dependencia (natural o que puede parecer natural) de un juez respecto a su Estado nacional. De esta manera, se procura evitar que los jueces sean a la vez jueces y partes en una controversia. En todos los ámbitos de impartición de la justicia internacional, el vínculo de nacionalidad que une a un juez internacional con su Estado nacional es visto como un elemento susceptible de violar el principio Nemo iudex in causa sua.

La cristalización del principio Nemo iudex in causa sua en los estatutos constitutivos de los tribunales internacionales demuestra, en primer lugar, la vigencia de este principio general en todos los mecanismos especializados de resolución de las controversias y, en segundo lugar, pone de manifiesto las similitudes y convergencias en la redacción de los artículos convencionales que se refieren a la independencia e imparcialidad de los jueces internacionales. Las mismas concordancias pueden observarse en la manera en la que los órganos judiciales internacionales aplican e interpretan el principio Nemo iudex in causa sua en la resolución concreta de las controversias que se les someten.

Artículos 3 y 17.

Artículo 4, párrafos 1 y 2, y artículo 10.

43 Artículo 12-1 del Estatuto del TPIY, artículo 11-1 del Estatuto del TPIR y artículo 35-7 del Estatuto de Roma.

44 En efecto, los jueces son designados entre los nacionales de cada una de las partes a la Convención europea de derechos humanos. Por lo tanto, la CEDH es necesariamente compuesta por un juez por Estado miembro. 


\section{La aplicación convergente del principio Nemo iudex in causa sua en la jurisprudencia de los tribunales internaciona- les}

El primer aspecto del principio Nemo iudex in causa sua -la imparcialidad de los jueces internacionales- ha sido invocado en varios casos de su jurisprudencia.

La CPJI se pronunció por primera vez respecto a la aplicación del principio Nemo iudex in causa sua en el caso Mosul (interpretación del artículo 3 del Tratado de Lausanne), en 1925. El origen de la controversia era una disputa territorial entre Turquía e Irak por la antigua provincia otomana de Mosul ${ }^{45}$. En virtud del artículo 3 del Tratado de Lausanne, las partes demandaron al Consejo de la Liga de Naciones arbitrar su controversia. El Consejo solicitó una opinión consultativa a la CPJI sobre varios problemas jurídicos suscitados por su litigio. La segunda pregunta presentada a la Corte concernía a las condiciones en las que el Consejo debía adoptar su decisión resolutoria del asunto. En particular, se trataba de saber si la decisión debía ser adoptada con la unanimidad o la mayoría de los miembros votantes y si en la votación podían participar representantes de los dos países, partes en la controversia. La Corte estimó que la decisión por la que el Consejo resolvía la controversia debía ser adoptada por unanimidad y que no había impedimento para la participación de representantes turcos e iraquíes en la votación. No obstante, la Corte afirmó que "la regla bien conocida según la cual nadie puede ser juez en su propio juicio” se aplica a las circunstancias. En consecuencia, la CPJI concluyó que los votos de los representantes de las dos partes en la controversia no debían ser contados para determinar si la decisión obtuvo la unanimidad de los votos en el Consejo ${ }^{46}$.

A su vez, la CIJ aplicó el principio Nemo iudex in causa sua en el caso Anglo-Iranian $\mathrm{Co}^{47}$. En dicho asunto, el juez de nacionalidad india Sir Benegal Raw declinó su competencia para participar en la resolución de la controversia porque había sido representante de la India en el Consejo de Seguridad de la ONU cuando éste adoptó una decisión que servía de antecedente para la resolución del caso en cuestión ${ }^{48}$.

45 Tanto Irak como Turquía pretendían ejercer su soberanía territorial sobre la provincia. La Liga de las Naciones había nombrado una comisión especial que después de entrevistar a la población de la provincia recomendó otorgar su administración al entonces Reino de Irak, bajo mandato británico.

46 CPJI (1925). Caso Mosul Boundary (opinión consultativa), párr. 95-98.

47 CIJ (1952). Anglo-Iranian Co. (Reino Unido c. Irán).

48 En particular, el Consejo de Seguridad había tenido que pronunciarse sobre la demanda de Gran Bretaña en contra de la inejecución, por parte de Irán, de las medidas cautelares pronunciadas por la CIJ en su sentencia previa. 
Posteriormente, en el caso Namibia ${ }^{49}$, la CIJ rechazó la demanda de Sudáfrica de recusar a los jueces Sir Zafrulla Khan, Padilla Nervo y Morozov debido a su previa participación en el caso en calidad de representantes de sus gobiernos nacionales ante la ONU. La Corte concluyó que la pasada actividad de los jueces como representantes de sus gobiernos no violaba el artículo 17 de su estatuto.

En el mismo sentido, en el caso Certain Phosphate Lands in Nauru ${ }^{50}$, uno de los jueces se recusó debido a su involucración previa en el caso. Otro juez de la CIJ estimó que no podía participar en la resolución de la controversia en el caso Arbitral Award of 31 july of $1989^{51}$, porque había sido árbitro en la sentencia arbitral que lo originó. A contrario, en el caso Rights of Nationals of the United States in Marocco ${ }^{52}$ participaron dos jueces que habían sido consejeros jurídicos de las partes en la controversia, en sus etapas anteriores.

La jurisprudencia de la CEDH respecto al principio Nemo iudex in causa sua se refiere a los criterios de aplicación del artículo 6 de la Convención europea de derechos humanos. Por lo tanto, se trata de la apreciación de la independencia e imparcialidad de los jueces internos y no internacionales. No obstante, dichos criterios merecen la atención porque fueron retomados por otros tribunales internacionales a través de la utilización de "referencias cruzadas" (cross-references).

Uno de los casos en los que la CEDH estableció con claridad el doble test de imparcialidad de un magistrado fue Piersack c. Bélgica ${ }^{53}$. En este caso, el Sr. Piersack alegaba la falta de imparcialidad de uno de los jueces del tribunal penal que lo condenó, en particular porque este juez había ejercido el cargo previo de Procurador. Según el demandante, dicho cargo le había permitido pre-juzgar en su caso y le había creado prejuicios susceptibles de afectar su imparcialidad. La CEDH estimó que la apreciación de la imparcialidad de un juez (interno) debe ser sujeta a un doble criterio. En primer lugar, se debe aplicar un criterio subjetivo, al determinar la convicción personal de un juez sobre un determinado caso y, en segundo lugar, se debe proceder a un examen objetivo para saber si las garantías a su imparcialidad son suficientes para excluir cualquier duda legítima al respecto ${ }^{54}$. Dicho en otros términos, no es necesario probar exclusivamente la imparcialidad subjetiva de un juez, sino que basta con alegar la existencia de

49 CIJ (1971). Legal Consequences for States of the Continued Presence of South Africa in Namibia (South West Africa) (opinión consultativa).

50 CIJ (1992). Certain Phosphate Lands in Nauru (Australia c. Nauru).

1 CIJ (1991). Arbitral Award of 31 july of 1989 (Guinea Bissau c. Senegal).

52 CIJ (1952). Rights of Nationals of the United States in Marocco (Francia c. Estados Unidos).

3 CEDH (1982). Piersack c. Bélgica.

54 En términos de la Corte: "Whilst impartiality normally denotes absence of prejudice or bias, its existence or otherwise can, notably under Article $6 \mathbb{S} 1$ (art. 6-1) of the Convention, be tested in various ways. A distinction can be drawn in this context between a subjective approach, that is endeavouring to ascertain the personal conviction of a given judge in a given case, and an objective approach, that is determining whether he offered guarantees sufficient to exclude any legitimate doubt in this respect" (Caso Piersack c. Bélgica, párr. 30). 
una apariencia suficientemente fundada de imparcialidad. En lo particular, la CEDH consideró que se trataba de una violación de este segundo criterio objetivo del test de imparcialidad. El juez, que previamente había fungido como Procurador, generó en el Sr. Piersack una duda legítima acerca de su imparcialidad. Consecuentemente, Bélgica fue condenada por una violación del artículo 6 de la Convención europea de derechos humanos. Este doble test de imparcialidad fue reiterado por la $\mathrm{CEDH}$ en varios casos de su jurisprudencia posterior ${ }^{55}$.

La jurisprudencia de la CEDH respecto a la imparcialidad de los jueces internos es literalmente seguida por la CIDH. Así por ejemplo, en el caso Herrera Ulloa ${ }^{56}$, la CIDH citó lo establecido en las sentencias Morris ${ }^{57}$ y Pabla $K Y^{58}$ de la CEDH, recordando el doble test de imparcialidad que se debe aplicar a los jueces nacionales. En términos de la Corte: "Primero, el tribunal debe carecer, de una manera subjetiva, de prejuicio personal. Segundo, también debe ser imparcial desde un punto de vista objetivo, es decir, debe ofrecer garantías suficientes para que no haya duda legítima al respecto. Bajo el análisis objetivo, se debe determinar si, aparte del comportamiento personal de los jueces, hay hechos averiguables que podrán suscitar dudas respecto de su imparcialidad. En este sentido, hasta las apariencias podrán tener cierta importancia. Lo que está en juego es la confianza que deben inspirar los tribunales a los ciudadanos en una sociedad democrática y, sobre todo, en las partes del caso" ${ }^{59}$. En lo particular, la CIDH consideró que los jueces de la Corte de casación de Costa Rica no habían reunido la exigencia de imparcialidad porque se habían pronunciado en una casación anterior respecto a gran parte del fondo del litigio del Sr. Herrera Ulloa, prejuzgando de esta manera de su asunto ${ }^{60}$. Las mismas consideraciones fueron expresadas por la CIDH en el caso Palamara

55 Así por ejemplo, en el caso Delcourt c. Bélgica, la CEDH examinó la función que cumple el Procurador General ante la Corte de casación belga. En efecto, según el derecho de este país, el Procurador podía estar presente en las deliberaciones de dicho tribunal sin posibilidad de votar. Aplicando el doble test de imparcialidad introducido en la sentencia Piersack, la CEDH concluyó que la presencia del Procurador en las deliberaciones no afectaba la independencia e imparcialidad de la Corte de casación belga. En el mismo sentido, en el caso Hauschildt v. Dinamarca (sentencia del 24 de mayo de 1989), el demandante ante la CEDH, Mogens Hauschildt, cuestionaba la imparcialidad de un juez, quien, después de haber dictado una serie de resoluciones decretando la prisión provisional del Sr. Hauschildt, formó parte del Tribunal de Copenhague que, junto con otros dos magistrados no profesionales, celebró la vista oral del procedimiento seguido en su contra y posteriormente dictó su sentencia condenatoria. La CEDH reiteró que la imparcialidad se debe valorar también con arreglo a un criterio objetivo que lleve a la seguridad de que reunía las garantías suficientes para excluir, a este respecto, cualquier duda legítima (Torre Blanco, Humberto. La imparcialidad objetiva en la jurisprudencia del Tribunal europeo de derechos humanos, pp. 7-10 [en línea] <http://congreso.us.es/cidc/Ponencias/judicial/HumbertoTorre.pdf> [consulta: 10 de junio del 2015].

56 CIDH (2004). Herrera Ulloa c. Costa Rica.

57 CEDH (2005). Steel and Morris vs the UK.

58 CEDH (2004). Pabla KY vs. Finlandia.

59 CIDH (2004). Herrera Ulloa c. Costa Rica, párr. 170.

60 CIDH (2004). Herrera Ulloa c. Costa Rica, párr. 171-175. 
Iribarne contra Chile ${ }^{61}$, respecto a la imparcialidad de los tribunales militares chilenos ${ }^{62}$. De la misma manera, la CIDH, en el caso Apitz Barbera y otros contra Venezuela ${ }^{63}$, citó lo establecido por la CEDH en los casos Pullar contra Reino Unido ${ }^{64}$, Fey contra Austria ${ }^{65}$, Daktaras contra Lituania ${ }^{66}$ y Piersack contra Bélgica para estimar que: "En cambio, la imparcialidad exige que el juez que interviene en una contienda particular se aproxime a los hechos de la causa careciendo, de manera subjetiva, de todo prejuicio y, asimismo, ofreciendo garantías suficientes de índole objetiva que permitan desterrar toda duda que el justiciable o la comunidad puedan albergar respecto de la ausencia de imparcialidad. La Corte Europea de Derechos Humanos ha explicado que la imparcialidad personal o subjetiva se presume a menos que exista prueba en contrario. Por su parte, la denominada prueba objetiva consiste en determinar si el juez cuestionado brindó elementos convincentes que permitan eliminar temores legítimos o fundadas sospechas de parcialidad sobre su persona. Ello puesto que el juez debe aparecer como actuando sin estar sujeto a influencia, aliciente, presión, amenaza o intromisión, directa o indirecta, sino única y exclusivamente conforme a -y movido por- el Derecho".

La jurisprudencia de la CEDH sobre la imparcialidad de los miembros de un tribunal fue retomada literalmente en una sentencia reciente de otra jurisdicción internacional. En efecto, el TPIY, en el caso Furundzija ${ }^{67}$, se basó en la jurisprudencia de la CEDH para aplicar el principio Nemo iudex in causa sua en el contexto específico de la justicia penal internacional.

En lo particular, se trataba de una alegación de imparcialidad en contra de la jueza Florence Mumba. Según los abogados del Sr. Furundzija, la jueza había defendido una causa política al proponer, en detrimento del acusado, que la violación se considerara como un crimen de guerra. En particular, los defensores del Sr. Furundzija alegaron que en su anterior puesto en la Comisión de la ONU sobre el estatus de las mujeres, la jueza

61 CIDH (2005). Palamara Iribarne contra Chile.

62 En particular, la CIDH estimó que: "La Corte considera que el derecho a ser juzgado por un juez o tribunal imparcial es una garantía fundamental del debido proceso. Es decir, se debe garantizar que el juez o tribunal, en el ejercicio de su función como juzgador, cuente con la mayor objetividad para enfrentar el juicio. Asimismo, la independencia del Poder Judicial frente a los demás poderes estatales es esencial para el ejercicio de la función judicial. La imparcialidad del tribunal implica que sus integrantes no tengan un interés directo, una posición tomada, una preferencia por alguna de las partes y que no se encuentren involucrados en la controversia. El juez o tribunal debe separarse de una causa sometida a su conocimiento cuando exista algún motivo o duda que vaya en desmedro de la integridad del tribunal como un órgano imparcial. En aras de salvaguardar la administración de justicia se debe asegurar que el juez se encuentre libre de todo prejuicio y que no exista temor alguno que ponga en duda el ejercicio de las funciones jurisdiccionales" (Sentencia Palamara Iribarne contra Chile, párr. 145-147).

$63 \mathrm{CIDH}$ (2008). Apitz Barbera y otros c. Venezuela.

64 CEDH (1996). Pullar vs. the UK.

65 CEDH (1993). Fey vs. Austria.

66 CEDH (2000). Daktaras vs. Lithuania.

67 TPIY (2000). Prosecutor vs. Furundzija, Cámara de apelaciones. 
Mumba había participado activamente en la elaboración de la Beijing Platform for Action. En dicho documento se proponía considerar la violación y otros crímenes de género como crímenes contra la humanidad. Según los abogados de la defensa, esta toma de posición previa respecto a uno de los problemas por resolver en el caso Furundzija, era susceptible de afectar la imparcialidad de la jueza Mumba. Además, según los defensores del acusado, debido a su participación en la Comisión de la ONU, la jueza Mumba se había reunido con tres grupos no gubernamentales que intervenían como amici curae en el caso Furundzija. Esta relación previa de la jueza con los amici también podría afectar su participación imparcial en el asunto ${ }^{68}$. Respecto a estas alegaciones, la Cámara de apelaciones del TPIY consideró que se debe apreciar no solamente la imparcialidad subjetiva de un juez, sino también las circunstancias que podrían crear una duda legítima respecto a su imparcialidad objetiva ${ }^{69}$. El TPIY consideró que un juez es imparcial si: 1) es demostrado que existe una imparcialidad actual (subjetiva) o si existe una apariencia inaceptable de imparcialidad (objetiva). Tal apariencia existe si el juez es parte del caso o si tiene intereses financieros o propietarios relacionados con su resolución, o si dicha resolución llevará a la obtención de una causa en la que el juez está involucrado junto con una de las partes. Bajo estas circunstancias, la recusación del juez debe ser automática; 2) las circunstancias llevarían a un observador razonable a dudar de la imparcialidad del juez ${ }^{70}$. Sobre la determinación de un "observador razonable”, el tribunal estimó que "una persona razonable debe ser una persona informada, con conocimiento acerca de todas las circunstancias relevantes de un caso, incluyendo las exigencias de independencia e imparcialidad (...) y tener conocimiento del hecho que la imparcialidad es uno de los deberes que los jueces prometen cumplir bajo juramento" ${ }^{71}$. En lo particular, la Cámara de apelaciones estimó que la relación de la jueza Mumba con algunos de los amici curae era demasiado distante para crear una duda razonable respecto a su imparcialidad. Además, el tribunal subrayó que su participación previa en los trabajos de la ONU acerca de los crímenes de género constituía una importante aportación para el desarrollo del Derecho internacional y, en vez de descalificar su labor como jueza en el caso Furundzija, la calificaba aún más ${ }^{72}$. Finalmente, el tribunal recordó que la imparcialidad de un juez internacional goza de una presunción muy difícil de refutar $^{73}$.

La cuestión de la imparcialidad de la jueza Florence Mumba fue también objeto de la demanda en el caso Talic ${ }^{74}$. En la opinión de los defensores del Sr. Talic, la participación

\footnotetext{
Miller, Burgh House Principles..., op.cit., p. 7.

TPIY (2000). Prosecutor vs. Furundzija, Cámara de apelaciones, párr. 189-191.

TPIY (2000). Prosecutor vs. Furundzija, Cámara de apelaciones, párr. 164-215 (apéndice IV).

TPIY (2000). Prosecutor vs. Furundzija, Cámara de apelaciones, párr. 190.

Miller, Burgh House Principles..., op.cit., pp. 7-8.

TPIY (2000). Prosecutor vs. Furundzija, Cámara de apelaciones, párr. 196-197.

TPIY (2000). Decision on the Application by Momir Talic for the Disqualification and Withdrawal of a Judge (Trial Chamber II).
} 
Revista Tribuna Internacional

Volumen $4 \cdot \mathrm{N}^{0} 7 \cdot 2015 \cdot$ pp. 129-151

ISSN 0719-210X (versión impresa)/ISSN 0719-482X (versión en línea)

previa de la jueza en el asunto Tadic, que trataba de hechos muy similares, era susceptible de crear una duda respecto a su imparcialidad. El TPIY rechazó la demanda, considerando que no se deben tomar en cuenta todas las apariencias de imparcialidad que las partes invocan, con el objetivo de lograr un juicio más favorable a su causa ${ }^{75}$.

De la misma manera, en el caso Kordic y Cerkez $^{76}$ el TPIY rechazó una demanda de recusación de los jueces Jorda y Riad debido a su participación previa en un caso que trataba de los mismos hechos.

Finalmente, en el caso Celebici $i^{77}$, el TPIY utilizó una "referencia cruzada" a la jurisprudencia de la CEDH para pronunciarse sobre una demanda de recusación por imparcialidad de la jueza Odio-Benito. Esta jueza había sido nominada recientemente a ocupar el cargo de Vice-Presidenta de Costa Rica, que en ese entonces era miembro no permanente del Consejo de Seguridad de la ONU. Los abogados de la defensa alegaron la incompatibilidad de dicha función con lo establecido en el artículo 16 del Estatuto del TPIY. Además, consideraron que dicha incompatibilidad era susceptible de crear una duda legítima respecto a su imparcialidad. Los miembros del TPIY rechazaron esta demanda y subrayaron que la jueza Odio-Benito había afirmado, en repetidas ocasiones, que no tomará posesión del cargo de Vice-Presidenta de su país antes de que se resuelva el caso pendiente ante el tribunal. Por lo tanto, no existía ni un cúmulo de funciones contrario con el artículo 16 del Estatuto del TPIY ni una apariencia justificada de imparcialidad $^{78}$.

La jurisprudencia del TPIR ofrece ejemplos muy interesantes de aplicación del principio Nemo iudex in causa sua en el contexto internacional. En efecto, a partir del caso Rutaganda ${ }^{79}$, el tribunal aceptó los criterios relativos a la apreciación de la imparcialidad de los jueces penales internacionales ya desarrollados en la jurisprudencia del TPIY. En particular, el tribunal utilizó una "referencia cruzada" al citar lo establecido al respecto en los casos Furundzija y Celebici, y estimó que el derecho a un tribunal independiente e imparcial forma parte del derecho a un debido proceso ${ }^{80}$.

La Cámara de apelaciones común de los dos tribunales penales ad hoc también aplicó el principio de imparcialidad de los jueces en el caso Renzaho ${ }^{81}$. En dicho caso, el acusado invocaba la imparcialidad de los jueces del TPIR debido a su participación previa en dos asuntos $^{82}$ que trataban de hechos idénticos a su causa. La Cámara de apelaciones citó lo

TPIY (2000). Decision on the Application by Momir Talic..., párr. 18.

TPIY (2004). Kordic y Cerkez.

TPIY (1998). Celebici.

Miller, Burgh House Principles..., op.cit., p. 6.

TPIR (2003). Georges A. N. Rutaganda vs. Prosecutor.

TPIR (2003). Georges A. N. Rutaganda vs. Prosecutor, párr. 38-42.

TPIR (2011). Tharcisse Renzaho vs. Prosecutor, Appeal Chamber.

TPIR (2008). Bagosora et al. vs. Prosecutor, Trial Chamber. 
establecido al respecto en su jurisprudencia previa, recordando que la imparcialidad de un tribunal es un requerimiento básico del derecho a un debido proceso, expresado en los artículos 19 y 20 del estatuto del tribunal y 15 (A) de sus reglas de procedimiento. El tribunal también citó lo establecido al respecto en los casos Furundzija y Celebici del TPIY y recordó el doble test de la CEDH acerca de la garantía de imparcialidad: se debe apreciar no sólo la imparcialidad subjetiva sino también la imparcialidad objetiva de un juez. El tribunal subrayó la presunción de imparcialidad de la que gozan todos los jueces, misma que puede ser difícilmente refutada. La carga de la prueba del contrario corresponde a la parte que alega la imparcialidad. Sobre la participación de los jueces internacionales en asuntos con hechos similares o idénticos, la Cámara de apelaciones afirmó que un juez penal no es descalificado por tener que pronunciarse sobre varios casos, originados por los mismos hechos ${ }^{83}$.

La cuestión de la imparcialidad de los jueces también fue invocada en el caso Hategekimana del 8 de mayo de $2012^{84}$. En dicho asunto, el acusado invocaba una apariencia de imparcialidad de los jueces de la Cámara de primera instancia del TPIR, debido a la participación de un jurista de dicho tribunal en un concurso de ensayos para jóvenes africanos. El ensayo que obtuvo el primer premio relataba los asesinatos cometidos por el acusado y contenía una ficción de la sentencia del TPIR en la que un juez lo reconocía culpable. Según su defensa, la participación de un jurista del tribunal en el jurado del concurso y su posterior participación en la redacción de la sentencia de la Cámara de primera instancia creaban una duda legítima respecto a la imparcialidad del órgano judicial. La Cámara de apelaciones rechazó su demanda, recordando que la adopción de las sentencias está a cargo exclusivo de los jueces y que los demás integrantes del tribunal no intervienen en dicha actividad. Además, la Cámara de apelaciones seńaló que según una jurisprudencia bien establecida en la materia, los jueces del tribunal se benefician de una presunción de imparcialidad que puede ser difícilmente refutada por la parte que la invoca ${ }^{85}$.

\section{Conclusiones}

En conclusión, podemos considerar que el principio Nemo iudex in causa sua constituye un principio fundamental del proceso judicial internacional. La cristalización convergente de los distintos aspectos de dicho principio en los estatutos constitutivos de las jurisdicciones internacionales demuestra su fundamental importancia para el ejercicio de la función judicial internacional. Además, su aplicación e interpretación concordante

\footnotetext{
TPIR (2011). Tharcisse Renzaho vs. Prosecutor, Appeal Chamber, párr. 20-22.

TPIR (2012). Ildelphonse Hategekimana vs. Prosecutor.

TPIR (2012). Ildelphonse Hategekimana vs. Prosecutor, párr. 13-22.
} 
Revista Tribuna Internacional

Volumen $4 \cdot \mathrm{N}^{0} 7 \cdot 2015 \cdot$ pp. 129-151

ISSN 0719-210X (versión impresa)/ISSN 0719-482X (versión en línea)

por parte de los tribunales internacionales manifiestan un acuerdo general (y universal) acerca de su tenor y alcances normativos. Tanto la consagración estatutaria de este principio como su interpretación y aplicación coherente en las sentencias de los tribunales internacionales demuestran que se trata de un principio procesal general y común a todas las esferas especializadas de impartición de la justicia internacional.

La utilización del principio Nemo iudex in causa sua como principio común a los tribunales internacionales presenta múltiples implicaciones para el funcionamiento de los mecanismos de impartición de justicia en el Derecho internacional.

En primer lugar, la utilización común de este principio pone de manifiesto la existencia de una armonización de distintos aspectos procesales de la impartición de la justicia internacional, en particular los relativos a las garantías del "debido proceso". La armonización en la aplicación de principios generales de naturaleza procesal manifiesta el desarrollo de estándares o modelos procesales compartidos por todos los tribunales internacionales. Así por ejemplo, muchos autores hablan del desarrollo de estándares interpretativos comunes ${ }^{86}$ a los tribunales internacionales o de la importancia del modelo de protección de los derechos humanos para todos los jueces internacionales ${ }^{87}$. En el mismo orden de ideas, la presencia de principios y normas comunes a los tribunales internacionales en materia de prueba y prácticas de indemnización ha permitido a Chester Brown introducir la hipótesis de la existencia de un "Common Law of International Adjudication" 88 . El análisis comparativo desarrollado en la presente investigación confirma que el principio Nemo iudex in causa sua podría ser considerado como parte de este "Derecho procesal común a los tribunales internacionales".

En segundo lugar, el análisis de la utilización del principio Nemo iudex in causa sua en la jurisprudencia de los tribunales internacionales, objeto de estudio, nos permite concluir que no existen divergencias en la aplicación e interpretación judicial de este principio. Dicho análisis corrobora que la aplicación e interpretación jurisprudencial del principio Nemo iudex in causa sua es sumamente coherente y convergente. Es de estimarse que la presencia de (al menos) un principio procesal común a los tribunales internacionales constituye una garantía para la coherencia y seguridad jurídica en la "adjudication" del Derecho internacional. La seguridad jurídica es un valor que todo Derecho debe poseer para poder cumplir eficazmente con una de sus funciones primarias: ordenar las relacio-

86 Matz-Luck, Nele. "Promoting the unity of International Law: Standard setting by international tribunals", en KöNIG, Doris, et. al. International Law: New challenges and the need for reforms?. Berlín, Springer, 2008, pp. 99-121.

87 CANivet, Guy. "Les influences croisées entre juridictions nationales et internationales, Eloge de la bénévolance des juges". Revue de science criminelle, 2005, p. 799; Slaughter, Anne-Marie. "Judicial Globalization”. Virginia Journal of International Law, vol. 40, 1999, p. 1103.

88 Brown, Chester. A Common Law of International Adjudication. Oxford, Oxford University Press, 2009. 
nes sociales ${ }^{89}$. Sin seguridad jurídica, los sujetos de un orden jurídico son incapaces de prever las consecuencias jurídicas de sus actos y, por ende, pueden perder su confianza en las instituciones y en las normas de dicho orden.

La existencia de una gran multitud de procedimientos judiciales inconexos y altamente especializados amenazaría el respeto de este valor jurídico en el ámbito internacional y expondría a los sujetos del Derecho internacional a múltiples controles judiciales, basados en distintas concepciones del proceso internacional que podrían conducir al establecimiento de derechos y obligaciones contrarias a dichos sujetos ${ }^{90}$, dependiendo de la institución judicial a la que se acuda para hacerlos valer. Por su parte, la aplicación uniformizada del principio Nemo iudex in causa sua garantiza a los sujetos del orden jurídico internacional el respeto de dos de los requerimientos más básicos y fundamentales de la impartición de la justicia: la independencia y la imparcialidad del juez internacional.

En tercer lugar, el análisis del principio Nemo iudex in causa sua permite constatar varias referencias cruzadas (cross-references, citations/références croisées) respecto a la interpretación y aplicación de dicho principio en la jurisprudencia internacional. Estas "referencias cruzadas" manifiestan el desarrollo de un "diálogo entre los jueces"- "diálogo entre las sentencias" o "diálogo jurisprudencial" sobre este principio general del Derecho. Podemos considerar que dichas referencias constituyen "influencias cruzadas" (crossinfluences, influences croisées) que crean vínculos funcionales, intelectuales y sobre todo jurisprudenciales entre los tribunales que recurren a esta práctica ${ }^{91}$. De esta manera, se operara una "fertilización" o "polinización cruzada" (cross-fertilization) entre las dos o más jurisdicciones involucradas en el diálogo judicial sobre el principio Nemo iudex in causa sua ${ }^{92}$. Este diálogo sin duda confirma la existencia de una coordinación informal entre los tribunales internacionales. Los jueces internacionales leen y citan las sentencias de otros jueces en materia de interpretación y aplicación del principio Nemo iudex in causa sua. Esto significaría que los jueces internacionales intentan coordinar su jurisprudencia en la materia, al menos de manera implícita. Los intentos conscientes (o inconscientes) de interrelacionar su actividad jurisprudencial demuestran que los tribunales internacionales no son completamente autónomos e independientes unos respecto a los otros. Es de considerarse que ninguna sentencia judicial internacional se elabora en una "autarquía" completa, por jueces narcisistas, introvertidos y encerrados en su

89 Fuller, Lon. The Morality of Law. New Have, Yale University Press, 1969.

90 Koskenniemi, Martti, Fragmentation of International Law: Topic (a): The Function and Scope of the Lex Specialis Rule and the Question of 'Self-Contained Regimes', p. 2 [en línea] <http://www.un.org/law/ilc/ sessions/55/fragmentation_outline.pdf> [consulta: 10 de junio del 2015].

91 Снanet, Christine. "Les influences croisées entre les juridictions nationales et les juridictions internationals". Les Cahiers de droit, vol. 51, 2010, p. 225.

92 Waters, Melissa. "Mediating norms and identity: The role of transnational judicial dialogue in creating and enforcing International Law”. Georgetown Law Journal, vol. 93, 2005, p. 490. 
Revista Tribuna Internacional

Volumen $4 \cdot \mathrm{N}^{0} 7 \cdot 2015 \cdot$ pp. 129-151

ISSN 0719-210X (versión impresa)/ISSN 0719-482X (versión en línea)

propio régimen jurídico especializado ${ }^{93}$. El análisis del principio Nemo iudex in causa sua demuestra que la interpretación y aplicación judicial del Derecho internacional es extrovertida, abierta a los intercambios e influenciada por las prácticas y la jurisprudencia de otros tribunales internacionales.

Por último, la utilización del principio Nemo iudex in causa sua en la jurisprudencia de los tribunales internacionales ratifica la importancia de los Principios Generales del Derecho para la conducta de los procedimientos judiciales internacionales. A pesar de que las normas procesales que los jueces internacionales están legitimados a aplicar son de carácter altamente específico y especializado, dichas normas no pueden regular la infinita variedad de situaciones fácticas que originan los litigios internacionales. Un juez internacional (y/o interno) necesita aplicar principios generales, de naturaleza procesal, que lo guíen en la impartición de la justicia, imperativo primero de una solución judicial de las controversias. Como indiqué anteriormente, el principio Nemo iudex in causa sua es inherente a las nociones mismas de Derecho, justicia y equidad que deben imperar en el arreglo judicial de los litigios. La naturaleza imprescindible de este Principio General del Derecho para el ejercicio de toda función judicial y su innegable presencia en la jurisprudencia de los tribunales internacionales especializados demuestra que los principios generales, fundamentales y estructurales del sistema jurídico internacional no están siendo afectados por la especialización de las normas y de los tribunales internacionales. Principios como Nemo iudex in causa sua siguen presentes en los regímenes normativos especializados. Su aplicación e interpretación jurisprudencial convergente preserva la coherencia de los overrarching principles (principios básicos) del orden jurídico internacional y evita su explosión en pequeñas partículas sin nada en común.

93 Canivet, "Les influences croisées...”, op. cit. 